\title{
Medical Imaging Informatics: Towards a Personalized Computational Patient
}

\section{N. Ayache \\ Inria (French Institute for Research in Computiational Scienes), Sophia Antipolis, France}

\section{Summary}

Medical Imaging Informatics has become a fast evolving

discipline at the crossing of Informatics, Computational Sciences,

and Medicine that is profoundly changing medical practices, for

the patients' benefit.

\section{Keywords}

Medical Imaging Informatics, personalized computational

patient, computational anatomy, computational medicine

Yearb Med Inform 2016; Suppl1:S8-9

http://dx.doi.org/10.15265//YS-2016-5002

Published online May 20, 2016
In 2002, my preface to the IMIA Yearbook was entitled "From Digital Anatomy to Virtual Scalpels and Image-Guided Therapy". It was announcing a revolution in medicine brought by the extensive use of medical image computing to better assist the diagnosis and therapy of the patient.

Today, the promised revolution is here: Medical images are omnipresent at the hospital, and Medical Imaging Informatics is required more than ever to exploit their flood of information. All around the world, medical image computing is used to extract the clinically relevant information from medical images, and to present this information in a way that is clinically useful to the physician. This is mainly done through the construction of a computational and personalized model of the patient.

Building a computational model of the human body requires dedicated algorithms that take into account a thorough knowledge of the human anatomy and physiology. Huge progress has been made during the last decades to describe and simulate the structure and functions of organs thanks to advanced mathematical, biological, physical, and chemical models of the living tissues at various scales from the nanoscopic (molecular) to microscopic (cellular), mesoscopic (tissue), and macroscopic (organic) scales.

Computational models of the human body rely on a set of parameters that allow, for instance, to specify the structure and function of organs. Generic models are based on average parameters estimated over a population. Confronted to in vivo anatomical and functional images and signals of a singular patient, those parameters are adjusted by efficient personalization

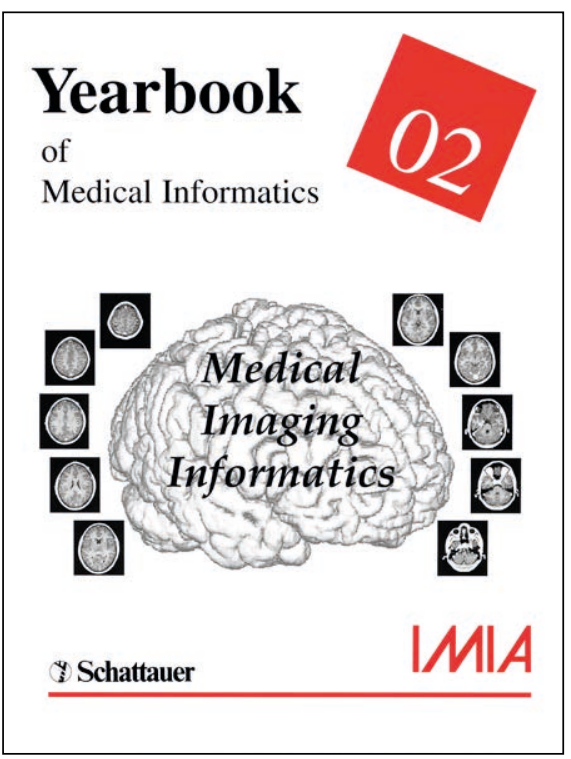

algorithms in order to reproduce more precisely the observed structures and functions leading to the personalized computational model of this particular patient.

The personalized computational model of the patient is then used to provide quantitative and objective measurements on the patient's condition to better assess the diagnosis. It is also used to predict a pathological evolution resulting in a better assessment of the prognosis. Finally, the computational model of the patient is extensively used to plan and simulate the effect of a therapy, in order to optimize its actual delivery. These three steps - computer-aided diagnosis, prognosis, and therapy - announce the fast development of the computational medicine at the service of the physician.

The tremendous progress of Medical Imaging Informatics also accompanies the evolution of normative and reactive medicine towards a more personalized, precise, preventive, and predictive medicine. This progress relies on numerous algorithmic advances in medical image analysis and inverse problem solving. It also relies on continuous advances in the modeling of human anatomy and physiology. It benefits from the improvement of medical image acquisition techniques, and from the introduction of new imaging modalities at various scales. It is supported by the regular 
increase of computer performances in terms of both computing power and memory storage. Finally it is boosted by the development of large databases of medical images made available through the Internet and by the introduction of large-scale machine learning methods.

In 2014, I had the privilege to be elected to the Chair of "Informatics and Computational Sciences" at the Collège de France. The inaugural lecture entitled "From Medical Images to the Digital Patient", the course on "The personalized digital patient: Images, Medicine and Informatics", and the associated invited lectures of 26 prominent colleagues are available on the web site of the Collège de France ${ }^{1}$.

These lectures reveal that the research in Medical Imaging Informatics is supported by a virtuous triangle. The first vertex represents a multidisciplinary academic research where informatics is associated with other sciences (mathematics, biology, physics, chemistry, anatomy, physiology, etc.). The second vertex represents a clinical partnership, essential to address relevant medical problems and validate the proposed solutions. The third vertex represents the industrial partnership required to transform the research prototypes into clinically approved products. These three worlds (academic, clinical and industrial) do not have rigid frontiers; actually their interactions are very stimulating and rich in terms of innovations.

A young and active international community of researchers supports the research in Medical Imaging Informatics. These researchers share a common vision and have developed a scientific society in 2004 called MICCAI (for Medical Image Computing and Computer Assisted Intervention). Born in 1998 with a few hundreds of researchers, its conference currently gathers each year on a different continent about 1,300 participants from 46 countries. About $40 \%$ of the participants are $\mathrm{PhD}$ students, which is a good sign of the vitality of this research community. Most of these young doctors find a position after their thesis, either in the academic, clinical, or industrial environment. An increasing number of them participate to the creation or development of start-up companies.
Medical Imaging Informatics has become a fast evolving discipline at the crossing of Informatics, Computational Sciences, and Medicine that is profoundly changing medical practices, for the patients' benefit!

\section{References}

1. Nicholas Ayache. Des images médicales au patient numérique [From medical imaging to the digital patient], Leçons inaugurales du Collège de France. Collège de France / Fayard, March 2015 (in French). http://books.openedition.org/cdf/4085

2. Nikos Paragios, Jim Duncan, and Nicholas Ayache. Handbook of Biomedical Imaging: Methodologies and Clinical Research. Springer, 2014

3. Website of the MICCAI Society: http://www. miccai.org/
Correspondence to:
Nicholas Ayache
Inria
Asclepios Project Team
2004 Route des Lucioles
06902 Sophia Antipolis
France
E-mail:nicholas.ayache@inria.fr

1 Courses and invited seminars available both in French and English languages at http://www.college-de-france.fr/site/ennicholas-ayache/ 